\title{
ESTIMATIONS OF EXPECTEDNESS AND POTENTIAL SURPRISE IN POSSIBILITY THEORY
}

\author{
Henri Prade \\ I.R.I.T.-C.N.R.S. \\ Universite Paul Sabatier \\ 118 route de Narbonne \\ 31062 Toulouse Cedex, France
}

\author{
Ronald R. Yager \\ Machine Intelligence Institute \\ Iona College \\ New Rochelle, N.Y. 10801, U.S.A.
}

\begin{abstract}
This note investigates how various ideas of "expectedness" can be captured in the framew'ork of possibility theory. Particularly, we are interested in trying to introduce estimates of the kind of lack of surprise expressed by people when saying "I would not be surprised that..." before an event takes place, or by saying "I knew it" after its realization. In possibility theory, a possibility distribution is supposed to model the relative levels of possibility of mutually exclusive alternatives in a set, or equivalently, the altematives are assumed to be rank-ordered according to their level of possibility to take place. Four basic set-functions associated with a possibility distribution, including standard possibility and necessity measures, are discussed from the point of view of what they estimate when applied to potential events. Extensions of these estimates based on the notions of Q-projection or OWA operators are proposed when only significant parts of the possibility distribution are retained in the evaluation. The case of partially-known possibility distributions is also considered. Some potential applications are outlined.
\end{abstract}

\section{1 - Introduction}

In case of incomplete knowledge, people facing a query like "is A (going to be) true ?", may answer it with a great variety of ways such that "I don't know", "it is not impossible", "it is quite possible", "I would not be surprised that A is true", "I am quite certain that A is true", etc., according to the actual state of knowledge and the query. Possibility theory (Zadeh, 1978) offers a framework for modelling uncertain or vague information by means of a possibility distribution. Such a distribution assesses the level of possibility of each possible value of a considered (single-valued) variable $x$, i.e. the elements of the domain of the variable $x$ are rank-ordered according to their relative possibility on the scale $[0,1]$. Then a possibility measure $\Pi$ is associated with the distribution, and $\Pi(A)$ estimates the consistency of the available knowledge with the statement " $A$ is true" (short for " $x$ is in $A$ is true"). A dual measure of necessity $N$ estimates the certainty of $A$ as the impossibility of "non $A$ ", namely $N(A)=\operatorname{Impos}(\bar{A})=$ $1-\Pi(\bar{A})$. Then $N($ non $A)$, the cerainty that $A$ is false, can be interpreted as a degree of surprise $S(A)=N($ non $A)=$ Impos(A) that $A$ is true. This corresponds exactly to the view developed by the English economist Shackle (1961) who worked out a non-probabilistic model of expectation, before the introduction of possibility theory. However this notion of surprise where $\Pi(A)=1-S(A)$ does not seem to correspond exactly to the intended meaning of a sentence such that "I would not be surprised that A is true", which rather expresses that "A true" is more than just possible (even with a high degree), and is not far to be somewhat certain; what is stated is a very strong kind of possibility.

In this note we investigate what estimates can be defined from a possibility distribution-based knowledge representation, in order to evaluate, in various ways, how much an event, such that " $x$ is in $A$ ", is expected to be true. The next section introduces four basic set functions defined from a possibility distribution, which are then extended using the notions of OWA operators, or of Q-projection, and also in the case of partially-defined possibility distributions.

\section{The Four Basic Set Functions in Possibility Theory}

Let $U$ be the domain of a single-valued variable $x$. In this note, $U$ is supposed to be finite for simplicity. A possibility distribution $\pi_{x}$ on $U$ is a function from $U$ to $[0,1]$ which constrains the possible values of $x$ according to the available information ; $\pi_{x}(u)=0$ means that $x=u$ is definitely impossible while $\pi_{x}(u)=1$ means that absolutely nothing prevents that $x=u$. A possibility distribution $\pi_{x}$ is said to be normalized iff $\exists u_{0} \in U$, $\pi_{x}\left(u_{0}\right)=1$, i.e. at least one value of $x$ in $U$ is completely possible, which is natural if $U$ is an exhaustive domain 
for $\mathrm{x} . \pi_{\mathrm{x}}$ can be viewed as a simple way of encoding a preference relation among the possible values of the variable $x$; the smaller $\pi_{x}(u)$, the more unexpected $x=u$ (or the less feasible $x=u$ ). It is assumed in the following that $\pi_{x}$ is normalized.

Given a possibility distribution $\pi_{x}$ and an event $A$, four basic estimates can be imagined which are in agreement with the ordinal nature of $\pi_{x}$; namely

- the possibility measure (Zadeh, 1978)

$$
\Pi_{\mathbf{X}}(\mathrm{A})=\max _{\mathrm{u} \in \mathrm{A}} \pi_{\mathbf{x}}(\mathrm{u}) \text {; }
$$

- the guaranteed possibility (Dubois and Prade, 1992)

$$
\Delta_{x}(A)=\min _{u \in A} \pi_{x}(u) ;
$$

and the similar evaluations for "non $A^{n}$, denoted $\bar{A}$, whose complements to 1 are taken in order to define meaningful quantities for $A$ ( $\pi_{x}$ should be normalized), namely

- the necessity measure

$$
N_{X}(A)=\min _{U \in A}\left(1-\pi_{X}(u)\right)=1-\Pi_{X}(\bar{A}) ;
$$

- the unguaranteed necessity

$$
\nabla_{X}(A)=\max _{u \in A}\left(1-\pi_{X}(u)\right)=1-\Delta_{X}(\bar{A}) .
$$

$\Pi_{\mathrm{X}}(\mathrm{A})$ estimates to what extent there exists a value $\mathrm{u}$ in $\mathrm{A}$ which is possible for $\mathrm{x}$, i.e. the consistency of the proposition " $x$ is in $A$ " with what is not unexpected according to the available information.

$\Delta_{x}(A)$ estimates to what extent all the values in $A$ are actually possible for $x$ according to what is known; any value in $A$ is at least possible for $x$ at the degree $\Delta_{x}(A)$; so $\Delta_{x}(A)$ expresses a guaranteed possibility since it is a minimum level over $A$.

$N_{x}$ (A) estimates to what extent all the values in $\bar{A}$ are impossible for $x$, or equivalently to what extent the value of $x$ is necessarily in $A$; any value in $\bar{A}$ is at most possible for $x$ at the degree $1-N_{x}(A)$.

$\nabla_{X}(A)$ estimates to what extent there exists a value $u$ in $\bar{A}$ which is impossible for $x$. It is a measure of unguaranteed necessity in favor of $A$ since we check the impossibility for $x$ of only one value in $\bar{A}$, and not the impossibility of all.

Clearly

$$
\begin{aligned}
\Delta_{\mathbf{X}}(\mathrm{A}) & \leq \Pi_{\mathbf{X}}(\mathrm{A}) \\
\mathrm{N}_{\mathbf{X}}(\mathrm{A}) & \leq \nabla_{\mathbf{X}}(\mathrm{A}) .
\end{aligned}
$$

Provided that $\pi_{x}$ is normalized, and that $\exists u \in U, \pi_{x}(u)=0$ (at the technical level, it is always possible to add an extra-element to $U$, if necessary, in order to satisfy this requirement), we have the stronger inequality (Dubois and Prade, 1992)

$$
\max \left(N_{x}(A), \Delta_{x}(A)\right) \leq \min \left(\Pi_{x}(A), \nabla_{x}(A)\right) .
$$

Thus $\Delta_{X}$ corresponds to a very strong possibility and $\nabla_{X}$ to a very weak necessity. Noticeably, $N_{X}$ and $\Delta_{X}$ are completely unrelated, as well as $\Pi_{x}$ and $\nabla_{x}$. When estimating the tendency of $A$ to contain the true value of $x$, we have indeed two complementary points of view, the extent to which values in $A$ are effectively possible, and the extent to which values out of $A$ are impossible. These two complementary evaluations may contribute to estimate our lack of surprise to have $A$ true.

The four measures enjoy the following characteristic properties (the subscript $\mathrm{x}$ is omitted in the following)

$$
\begin{aligned}
\Pi(A \cup B) & =\max (\Pi(A), \Pi(B)) \\
\Delta(A \cup B) & =\min (\Delta(A), \Delta(B)) ; \\
N(A \cap B) & =\min (N(A), N(B)) \\
\nabla(A \cap B) & =\max (\nabla(A), \nabla(B)) .
\end{aligned}
$$

Thus $\Pi$ and $N$ are monotonically increasing with respect to set inclusion, while $\Delta$ and $\nabla$ are decreasing. 
The interval $[\Delta(A), \Pi(A)]$ characterizes the amplitude of the variation of the levels of possibility among the values in $A$, the interval $[N(A), \nabla(A)]=1-[\Delta(\bar{A}), \Pi(\bar{A})]$ the amplitude of the variations in $\bar{A}$. We can then symbolically write $[N(A), \nabla(A)]=[N, \nabla](A)$ and $[\Delta(A), \Pi(A)]=[\Delta, \Pi](A)$; then we have

and (8)-(9)-(10)-(11) become

$$
[N, \nabla](A)=1-[\Delta, \Pi](\bar{A})
$$

$$
\begin{aligned}
{[\Delta, \Pi](A \cup B) } & =m M([\Delta, \Pi](A),[\Delta, \Pi](B)) \\
{[N, \nabla](A \cap B) } & =m M([N, \nabla](A),[N, \nabla](B))
\end{aligned}
$$

with $\operatorname{mM}([a, b],[c, d])=[\min (a, c), \max (b, d)]$, and then $1-m M([a, b],[c, d])=\operatorname{mM}(1-[a, b], 1-[c, d])$. Note that $\mathrm{mM}([\mathrm{a}, \mathrm{b}],[\mathrm{c}, \mathrm{d}])=$ convex_hull$([\mathrm{a}, \mathrm{b}] \cup[\mathrm{c}, \mathrm{d}])$.

We now discuss what measures of expectedness and surprise are, and we introduce generalizations of the set functions $\Pi, \Delta, N, \nabla$ based on the notions of OWA operators, or of Q-projection, in order to build intermediary estimates which may be used as estimates of how much an event $A$ is expected to be true.

\section{3 - Measures of Expectedness and Surprise}

In this section we shall introduce some formal mechanism for capturing the concepts of "expectedness" and "surprise" associated with a set, based upon the assumption of some possibility distribution.

Assume we are concerned about John's height. Then a possibility distribution would be induced by the knowledge that John is "tall". In this situation if it was found that John's height is six-feet seven inches one would not be surprised and would even have expected an answer like that. We shall in the following suggest some formal methods for capturing a measure of these concepts.

Assume we have a variable $x$ which induces a possibility distribution $\pi_{x}$ on $U$. Let $A$ be a crisp subset of $U$. We shall let $\operatorname{Exp}(A)$ measure the degree of expectedness of $A$ based upon $\pi$. We shall define this measure as the truth of the proposition

$$
\text { "most of the elements not in A are not possible". }
$$

We can more formally express this partial inclusion of $\bar{A}$ into the fuzzy set of values of $U$ which are rather impossible, as

$$
\operatorname{Exp}(A)=\text { most }_{u \in} \bar{A}[1-\pi(u)]
$$

where 'most $t_{u}$ ' refers to the proportion of elements in $\bar{A}$ whose degree of possibility should be low. In this section and in the next one, we shall propose two slightly different ways of precisely defining this formal expression, either using OWA operators or Q-projections. Let us first consider a special extreme case of 'most' : "all". In this case

$$
\operatorname{Exp}(A)=\min _{u \in} \bar{A}[1-\pi(u)] .
$$

Thus this extreme definition becomes what we previously called the necessity measure. Thus the extreme of expectedness is necessity.

In order to evaluate expectedness in the general case, we can use the concept of OWA operators introduced by Yager (1988), i.e.

$$
\operatorname{Exp}(A)=O W A_{u \in \bar{A}}[1-\pi(u)]
$$

Let $\bar{A}=\left\{u_{1}, \ldots, u_{r}\right\}$. Let $a_{i}=1-\pi\left(u_{i}\right)$. Let $\left\{\omega_{1}, \ldots, \omega_{r}\right\}$ be a set of weights such that

1) $\forall \mathrm{i}, \omega_{\mathrm{i}} \in[0,1]$;

2) $\Sigma_{\mathrm{i}} \omega_{\mathrm{i}}=1$;

then OWA $\left(a_{1}, \ldots, a_{r}\right)=\Sigma_{i} \omega_{i} \cdot b_{i}$, where $b_{i}$ is the ith largest of the $a_{j}$. Two extreme cases of weights are worth noting. Taken $\omega_{\mathrm{r}}=1$ (and then all others are zero), we get : 


$$
\operatorname{OWA}\left(a_{1}, \ldots, a_{\mathrm{r}}\right)=b_{\mathrm{T}}=\min _{\mathrm{i}} a_{\mathrm{i}}=\min _{\mathrm{u} \in \overline{\mathrm{A}}}[1-\pi(\mathrm{u})],
$$

i.e. the necessity measure of $A$. When $\omega_{1}=1$ (and then all others are zero), we get

$$
\operatorname{OWA}\left(a_{1}, \ldots, a_{r}\right)=b_{1}=\max _{i} a_{i}=\max _{u \in A}[1-\pi(u)],
$$

which is what we previously called the unguaranteed necessity. When $\omega_{j}=1 / k$ for $i=1, k$ with $k \leq r$, we compute the average of the $k$ largest levels of impossibility $a_{i}=1-\pi\left(u_{i}\right)$. Following Yager (1988)'s discussion, we can express "most" by an appropriate selection of weights.

We need now introduce a formal definition for "surprise of $A^{n}$ given a possibility distribution. We denote Sur(A) as the measure of surprise and define it as the truth of the proposition

"most of the elements in A are not possible".

We can more formally express this as

$$
\operatorname{Sur}(A)=\operatorname{most}_{u \in A}[1-\pi(u)] .
$$

As we can see we have $\operatorname{Sur}(A)=\operatorname{Exp}(\bar{A})$, which expresses that $A$ is surprising if non-A is expected. However we do not have $\operatorname{Sur}(A)=1-\operatorname{Exp}(A)$ in the same time (i.e. "A is surprising" is different from " $A$ is unexpected" in our model). Clearly these two understandings of $\operatorname{Sur}(A)$ would be equivalent in a probabilistic model. Again considering the special case where "most" is replaced by "all" we get

$$
\operatorname{Sur}(A)=\min _{u \in A}[1-\pi(u)] .
$$

This special case can be further simplified so that

$$
\operatorname{Sur}(A)=1-\max _{\mathrm{u} \in A} \pi(\mathrm{u})=1-\Pi(\mathrm{A})
$$

which corresponds to Shackle (1961)'s definition.

Considering the more general case of surprise (with "most" in place of "all"), we can use OWA operators to implement the formal expression by appropriate selection of the weights. At the extreme when $\omega_{S}=1$, with $A=$ $\left\{u_{r+1}, \ldots, u_{S}\right\}$, we get $\operatorname{Sur}(A)=\min _{u}(1-\pi(u))=1-\Pi(A)$, while when $\omega_{r+1}=1$

$$
\operatorname{Sur}(A)=\max _{u}(1-\pi(u))=1-\min _{u \in A} \pi(u)=1-\Delta(A) .
$$

We can further observe that if one considers the negation of "most" as "at_least_a_few", then

$$
\operatorname{Sur}(A)=1-\text { at_least_a_few }_{u \in A}[\pi(u)]
$$

where at_least_a_few corresponds to an ordered weighted average OWA' related to the one defining $\operatorname{Sur}(A)=$ OWA $_{u \in A}[1-\pi(u)]$ in the following way. $\operatorname{Sur}(A)=O W A\left(1-\pi\left(u_{r+1}\right), \ldots, 1-\pi\left(u_{s}\right)\right)=\Sigma_{i} \omega_{i} \cdot b_{i}^{\prime}$ where $b_{i}^{\prime}$ is the ith largest of the $1-\pi\left(u_{j}\right)$. Then $\operatorname{Sur}(A)=\Sigma_{i} \omega_{i}-\Sigma_{i} \omega_{j}\left(1-b_{i}^{\prime}\right)=1-$ OWA $^{\prime}\left(\pi\left(u_{r+1}\right), \ldots, \pi\left(u_{s}\right)\right)=$ $1-\Sigma_{\mathrm{i}} \omega_{\mathrm{i}} \cdot c_{\mathrm{i}}$ where $c_{\mathrm{i}}$ is the ith smallest of the $\pi\left(\mathrm{u}_{\mathrm{j}}\right)$.

Remark : Extension to belief structures

We shall here briefly suggest the extension of the preceeding ideas to the case in which our basic knowledge is a belief structure of the type introduced by Shafer (1976). Assume we have a belief structure consisting of the focal elements $B_{1}, \ldots, B_{n}$ with weights $m\left(B_{\mathfrak{i}}\right)$ (and $\left.\Sigma_{i} m\left(B_{\mathfrak{i}}\right)=1\right)$. We can define the degree of amazement associated with the subset $A$ as

where

$$
\operatorname{Amaze}(A)=\Sigma_{i} \operatorname{Sur}\left(A \mid B_{\mathfrak{i}}\right) \cdot m\left(B_{\mathfrak{i}}\right)
$$

$$
\operatorname{Sur}\left(A \mid B_{i}\right)=\operatorname{most}_{u \in A}\left[1-\mu_{B_{i}}(u)\right]
$$


and $\mu_{B_{i}}$ is the characteristic function of $B_{i}$. We can define the degree of anticipation associated with $A$ as

where

$$
\text { Anticipate }(\mathrm{A})=\Sigma_{\mathrm{i}} \operatorname{Exp}\left(\mathrm{A} \mid \mathrm{B}_{\mathbf{i}}\right) \cdot \mathrm{m}\left(\mathrm{B}_{\mathbf{i}}\right)
$$

$$
\operatorname{Exp}\left(A \mid B_{i}\right)=\text { most }_{u \in} \bar{A}\left[1-\mu_{B_{i}}(u)\right]
$$

\section{4 - Generalizations Based on Q-Projection}

As established in the preceeding sections, we have shown that $\Pi, \Delta$ (resp. $: N, \nabla$ ) are closely related to the concepts of surprise and expectedness. These concepts actually being related to the extremes of these measures. Crucial to the determination of the measures of surprise and expectedness are evaluations based upon quantifiers such as "most" lying being the extremes "for all" and "there exists" (corresponding to min and max operations). In the previous section we have suggested the use of OWA operators to implement these soft quantifiers. In this section we shall suggest an alternative approach to the kinds of evaluations necessary. This approach is based upon the notion of Qprojection (Yager, 1985). We only consider the case of non-fuzzy quantifiers where $Q$ is a quantifier of the type "at least $\mathrm{r} / \mathrm{k}$ " for simplicity. We define each Q-projection in terms of a median operator, which has some notation advantage for expressing Q-projection. In the following we shall first define the concept of $Q$-possibility of $\mathrm{A}$. In the ordinary measure of possibility we have $Q=$ "at least one" (thus $A$ is possible if just one element in $A$ is possible), while for Q-possibility measure of the type discussed here, we have $Q=$ "at least $r / k$ ", where $k$ is the cardinality of the set $A$. In this more general setting we are saying that $A$ is $Q$-possible if at least $r / k$ of the elements in $A$ are possible. We further note that if we define "most" by the appropriate selection of some value $r$ as explained below, we have

$$
\operatorname{Sur}(A)=1-\text { Q-possibility(A). }
$$

Let $A=\left\{u_{1}, \ldots, u_{k}\right\}$ be the finite subset of $U$ on which we want to estimate to what extent a given number (or a given proportion) of values of $A$ are possible. This number or proportion can be translated into a $k$-tuple of the form $\mathrm{Q}=(1, \ldots, 1,0, \ldots, 0)$ where $\mathrm{k}=|\mathrm{A}|$, and where the number of ' 1 ' in the tuple representing $\mathrm{Q}$ is $\mathrm{r}$. Then the $Q$ possibility of $A$, denoted by $Q(A)$, is defined by

$$
Q(A)=\operatorname{median}\left(\left\{\pi\left(u_{1}\right), \ldots, \pi\left(u_{k}\right)\right\} \cup \bar{Q} \cup(1\}\right)
$$

where $Q$ denotes the complement of $Q$. Indeed, $Q(A)$ is obtained as the median of a set of $2 k+1$ elements made of $k$ $-r+1$ elements equal to ' 1 ', of the $k$ values $\pi\left(u_{1}\right), \ldots, \pi\left(u_{k}\right)$, and of $r$ values equal to 0 . Thus, $Q(A)$ is equal to the $(\mathrm{k}+1)$ th value when the $2 \mathrm{k}+1$ elements are ranked in decreasing order, i.e. the rth value in the set $\left[\pi\left(u_{1}\right), \ldots\right.$, $\left.\pi\left(u_{k}\right)\right]$, once these degrees are decreasingly ordered. Clearly $Q=(1,0, \ldots, 0)($ with $(k-1)$ ' 0$)$ gives back $Q(A)=$ $\Pi(A)$, while $Q=(1,1, \ldots, 1)$ (with $k$ ' 1 ) yields $Q(A)=\Delta(A)$. Clearly, in any case

$$
\mathrm{Q}(\mathrm{A}) \in[\Delta, \Pi](\mathrm{A}) \text {. }
$$

It can be shown (see Prade (1990) for instance) that the Q-possibility of A is nothing but the possibility measure that the number of possible elements (according to $\pi$ ) is at least $r$, computed from the possibility distribution representing the more or less possible values of the cardinality of the fuzzy subset of A made of the elements which are rather possible.

By duality, quantities of the form $1-Q^{\prime}(\bar{A})$ can be introduced. We have

$$
\begin{aligned}
1-Q^{\prime}(\bar{A}) & =1-\operatorname{median}\left(\left\{\pi\left(u_{1}^{\prime}\right), \ldots, \pi\left(u_{k^{\prime}}^{\prime}\right)\right\} \cup \bar{Q}^{\prime} \cup\{1\}\right) \\
& =\operatorname{median}\left(\left\{1-\pi\left(u_{1}^{\prime}\right), \ldots, 1-\pi\left(u_{k^{\prime}}^{\prime}\right)\right\} \cup Q^{\prime} \cup\{0\}\right)
\end{aligned}
$$

where $\bar{A}=\left\{u_{1}^{\prime}, u_{2}^{\prime}, \ldots, u_{k^{\prime}}^{\prime}\right\}, k^{\prime}=|\bar{A}|$, and $Q^{\prime}$ is a $k^{\prime}$-tuple of ' 1 ' and ' 0 '. When $Q^{\prime}=(1,0, \ldots, 0)$, we recover $N(A)=$ $1-Q^{\prime}(\bar{A})$, and when $Q^{\prime}=(1,1, \ldots, 1)$, we get $\nabla(A)=1-Q^{\prime}(\bar{A})$. When "most" of the values in $A$ are highly possible, or when only few values outside $A$ are possible (i.e. equivalently, "most" of the values in $\bar{A}$ are impossible), which can be estimated using respectively $Q(A)$ (with $r$ "close" to $k$ ), and $1-Q$ ' $(\bar{A})$ where $Q$ ' models "few" (the number of ' 1 ' in $Q$ ' is small), we may consider that this is the kind of situation where we would expect 
that $A$ is true. Unfortunately, $Q$ does not enjoy a decomposability property with respect to the union of subsets in the general case, as $\Pi$ and $\Delta$ do.

We may conclude that A should be true either by checking, on a completely known possibility distribution, that (at least) most values outside $A$ are impossible for instance, or from the computation of approximations of $[\Delta, \Pi](A)$ and $[\mathrm{N}, \nabla](\mathrm{A})$ on the basis of a partially-known possibility distribution, as explained below.

\section{5 - Estimations Based on Partially-Known Possibility Distributions}

By a partially known possibility distribution, we mean that for each element $u$ of $U$, the degree of possibility $\pi_{x}(u)$ is only known to belong to an interval $\left[\pi_{x}(u), \pi^{+} x(u)\right]$. The upper bound $\pi^{+} x$ is normalized on $U$ since $\pi_{x}$ is supposed to be normalized, while $\pi^{-}$is not necessarily normalized.

Then, the following bounds can be computed

$$
\begin{aligned}
& \Pi^{+}(A)=\max _{u \in A} \pi_{x}^{+}(u) \geq \Pi(A) \geq \max _{u \in A} \pi_{x}(u)=\Pi^{-}(A) \\
& N^{+}(A)=\min _{u \in A}\left(1-\pi_{x}^{+}(u)\right) \leq N(A) \leq \min _{u \in A}\left(1-\pi_{x}^{-}(u)\right)=N^{-}(A) \\
& \Delta^{+}(A)=\min _{u \in A} \pi^{+}(u) \geq \Delta(A) \geq \min _{u \in A} \pi_{x}(u)=\Delta^{-}(A) \\
& \nabla^{+}(A)=\max _{u \in A}\left(1-\pi^{+}{ }_{x}(u)\right) \leq \nabla(A) \leq \max _{u \in A}\left(1-\pi_{x}(u)\right)=\nabla^{-}(A) .
\end{aligned}
$$

In other words we have inner and outer approximations of $[\Delta, \Pi]$ and $[N, \nabla]$, namely

$$
\begin{aligned}
& \forall A,\left[\Delta^{+}, \Pi^{-}\right](A) \subseteq[\Delta, \Pi](A) \subseteq\left[\Delta^{-}, \Pi^{+}\right](A) \\
& \forall A,\left[N^{-}, \nabla^{+}\right](A) \subseteq[N, \nabla](A) \subseteq\left[N^{+}, \nabla^{-}\right](A)
\end{aligned}
$$

However $\left[\Delta^{+}, \Pi^{-}\right](A)$ may be empty if it happens that $\Delta^{+}(A)>\Pi^{-}(A)$, as well as $\left[N^{-}, \nabla^{+}\right](A)$ if $N^{-}(A)>$ $\nabla^{+}(A)$. A particular case which is worth considering is when $\exists V \subseteq U, \forall u \in V, \pi^{-} x(u)=\pi^{+} x(u)=\pi_{x}(u)$ and $\forall u$ $\in U-V, \pi_{X}(U)=0, \pi^{+}{ }_{x}(U)=1$, i.e. $\pi_{x}$ is perfectly known on a part of $U$ and completely unknown elsewhere. Then the lower bound of $N(A)$

$$
N^{-}(A)=\min _{u \in A \cap V}\left(1-\pi_{x}(u)\right)=N(A \cup V) \geq N(A)
$$

(while $\mathrm{N}^{+}(A)=0$ as soon as $\mathrm{V} \geqq \bar{A}$ ) is a good candidate for estimating a beginning of certainty in favor of $A$. Indeed, $N^{-}(A)=N(A \cup \nabla)$ corresponds to the certainty in favor of a set less specific than $A$, but which contains $A$. Note that $N(A) \geq N(A \cap V)=\min (N(A \cup \bar{V}), N(V))$ where $N(V)$ is totally unknown, since $\pi_{x}$ is only supposed to be known on $V$. Then $N^{-}(A)=N(A \cup V)$ is a good approximation of the certainty of $A$ with respect to the available information. Moreover if, together with $N^{-}(A)>0, \Delta^{+}(A)=\min _{u \in A \cap V} \pi_{x}(U)=\Delta(A \cap V) \leq \Pi^{-}(A)=$ $\max _{\mathrm{u} \in \mathrm{A} \cap \mathrm{V}}=\Pi(\mathrm{A} \cap \mathrm{V}) \leq \Pi(\mathrm{A})$ is large enough, we would not be surprised that $A$ turns to be true.

\section{6 - Potential Applications}

Although this note is basically oriented towards the formalization of the concepts of expectedness and surprise in the framework of possibility theory, let us briefly outline some potential applications.

A first use we may think of is the representation of decision rules of the kind "if $A$ is expected then do..." which is a soft and more realistic version of the rule "if $A$ is certain then do...".

Another use might be in information systems where we want to rank the items according to what extent they can be expected to satisfy the request. This might be of interest particularly if the set of items which more or less certainly satisfy the request is empty and the set of items which satisfy it only possibly is too large. 
Clearly, it is not only important to be able to represent incomplete, uncertain, vague states of knowledge, but also to understand what a model offers for modelling expectation. This may be important in knowledge-based systems for instance for representing the state of knowledge of the user and deciding what explanation has to be provided to him/her (usually what is expected has not to be explained and what is surprising has to be explained).

Another issue that our future work in this area will focus on is possibility distribution generation based upon surprise and expectedness qualification. Consider a proposition like "I expect (at degree $\alpha$ ) that John will be late". This proposition can be seen to induce a possibility distributions $\pi$, over John's arrival time. In particular we see that this requires the solution of an equation of the type $\alpha=\operatorname{Exp}[A / \pi]$. In a similar fashion propositions like "I would be surprised (at the degree $\alpha$ ) if John is early" or "I would not be surprised (at the degree $\beta$ ) if John is late" can be seen to induce possibility distributions. The ability to generate possibility distributions from propositions of the above type would provide an interesting tool in knowledge representation.

\section{7 - Concluding Remarks}

In this note we have investigated all the estimates which can be attached to a non-fuzzy event $A$, when the available knowledge is modelled by a possibility distribution (even if this distribution is partially specified). The role of four basic measures has been emphasized, two of them define an interval related to the estimation of the idea of possibility, while the two others define another interval related to the idea of necessity or certainty. The characteristic properties of these intervals have been laid bare. Other quantities, which generalize the previous ones in various ways, have been introduced. The appropriateness of these different degrees for estimating how much an event can be expected to be true, how much its occurrence is not surprising, has been discussed. All these measures could be extended to fuzzy events A.

\section{References}

Dubois D., Prade H. (1992) Possibility theory as a basis for preference propagation in automated reasoning. Proc. of the 1st IEEE Inter. Conf. on Fuzzy Systems (FUZZ-IEEE'92), San Diego, CA, March 8-12, 821-832.

Prade H. (1990) A two-layer fuzzy pattern matching procedure for the evaluation of conditions involving vague quantifiers. J. of Intelligent and Robotic Systems, 3, 93-101.

Shackle G.L.S. (1961) Decision, Order and Time in Human Affairs. (2nd edition) Cambridge University Press.

Shafer G. (1976) A Mathematical Theory of Evidence. Princeton University Press, Princeton, NJ..

Yager R.R. (1985) Q-projections of possibility distributions. IEEE Trans. on Systems, Man and Cybernetics, 15, 775-777.

Yager R.R. (1988) On ordered weighted averaging aggregation operators in multi-criteria decision making. IEEE Trans. on Systems, Man and Cybernetics, 18, 183-190.

Zadeh L.A. (1978) Fuzzy sets as a basis for a theory of possibility. Fuzzy Sets and Systems, 1, 3-28. 